\title{
A Condition-Based Failure-Prediction and Processing-Scheme for Preventive Maintenance
}

\author{
S. K. Yang
}

\begin{abstract}
Preventive maintenance (PM) is an effective approach for reliability enhancement. Time-based and condition-based maintenance are two major approaches for PM. In contrast, condition-based maintenance can be a better and more cost-effective type of maintenance than time-based maintenance. However, irrespective of the approach adopted for PM, whether a failure can be detected early or even predicted is the key point. This paper presents a failure prediction method for PM by state estimation using the Kalman filter. To improve preventive maintenance, this study uses a hybrid Petri-net modeling method coupled with fault-tree analysis and Kalman filtering to perform failure prediction and processing. A Petri net arrangement, viz, early failure detection and isolation arrangement (EFDIA), is used; it facilitates alarm, early failure detection, fault isolation, event count, system-state description, and automatic shutdown or regulation. These functions are very useful for health-monitoring and preventive-maintenance of a system. This study implements EFDIA to an application-specific integrated circuit on a Xilinx Demonstration Board. A condition-monitoring system of a thermal power plant is used as an example to demonstrate the proposed scheme. Linking the Kalman filter to the EFDIA Petri net, a condition-based failure prediction and processing scheme has been completed for preventive maintenance.

This paper presents a failure prediction and processing scheme for PM via the thermal power-plant example, by using a hybrid Petri net modeling method endowed with fault-tree analysis and Kalman filtering. The FPN (Petri net dealing with system failure) has to be constructed beforehand. The next step is to obtain control charts for all fault places in the FPN in order to prescribe thresholds and increment times for every step in Kalman prediction. Afterwards, the system model of each place in the FPN must be derived to perform Kalman filtering. With these prerequisites, this method can be applied to any system. The proposed Petri net approach not only can achieve early failure detection and isolation for fault diagnosis but also facilitates event count, system state description, and automatic shutdown or regulation. These capabilities are very useful for health monitoring and PM of a system. Since the triggering signal of $S_{i}$ place of the EFDIA in Section IV $\left(S_{i}\right.$ is a place for the Kalman-predicted indicator value of the sensing signal for the Petri net dealing with system failure) indicates that subsystem \# $i$ performance is going to reach the prescribed failure threshold, the signal can be provided via the Kalman filtering method in Section III. Linking the Kalman filter to the EFDIA Petri net, a condition-based failure prediction and processing scheme has been completed for preventive maintenance.
\end{abstract}

Manuscript received August 15, 2001; revised February 4, and May 24, 2002. This work was supported by the National Science Council in Taiwan under Grant NSC 87-TPC-E009-005 and Grant NSC 89-2213-E167-015. Responsible Editor: W-T. K. Chien.

S. K. Yang is with the Department of Mechanical Engineering, National Chin Yi Institute of Technology, Taichung 411, TAIWAN-R.O.C. (e-mail SKYang@chinyi.ncit.edu.tw).

Digital Object Identifier 10.1109/TR.2003.816402
Index Terms-ASIC (application-specific integrated circuit), failure prediction, fault-tree analysis, kalman filter, petri net, preventive maintenance, reliability analysis.

\section{ACRONYMS}

ARMA auto-regressive and moving average

ASFM automatic shutdown-or-feedback mechanism

ASIC application-specific integrated circuit

CM corrective maintenance

EFDIA early failure detection-and-isolation arrangement

FPGA field-programmable gate array

FPN Petri net dealing with system failure

IM improvement maintenance

PM preventive maintenance

$s \quad$ statistical(ly)

\section{NOTATION}

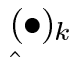

the value of $(\bullet)$ at time $k \cdot T$

estimate of $(\cdot)$ at time $a \cdot T$ based on all known information about the process up to time $b \cdot T$

$\mathbf{B}_{k} \quad$ coefficient matrix for the input term of a discrete state equation

$E[\bullet] \quad s$-expected value of the variable inside the [ ]

$\mathbf{H}_{k} \quad$ matrix for the ideal (noiseless) connection between the measurement and the state vector

$K_{k} \quad$ Kalman gain

$P_{k / k-1} \quad$ estimation-error covariance matrix

$Q_{k} \quad$ covariance matrices for disturbance

$R_{k} \quad$ covariance matrices for noise

$t \quad$ time variable

$T \quad$ increment time for every step in Kalman prediction

$U_{k} \quad$ control input of a discrete-state equation

$V_{k} \quad$ noise, measurement error vector (assumed to be a white sequence with known covariance)

$\mathbf{W}_{k} \quad$ disturbance, system stochastic input vector (assumed to be a white sequence with known covariance and having zero cross-correlation with the $V_{k}$ sequence)

$\begin{array}{ll}\mathbf{X}_{k} & \text { system-state vector } \\ \mathbf{Y}_{k} & \text { system-output vector } \\ \mathbf{Z}_{k} & \text { output-measurement vector } \\ \phi_{k} & \text { matrix relating } X_{k} \text { to } X_{k+1}, \text { in the absence of a } \\ & \text { forcing function (the state transition matrix if } X_{k} \text { is } \\ & \text { sampled from a continuous process) }\end{array}$

${ }^{1}$ The singular and plural of an acronym are always spelled the same. 


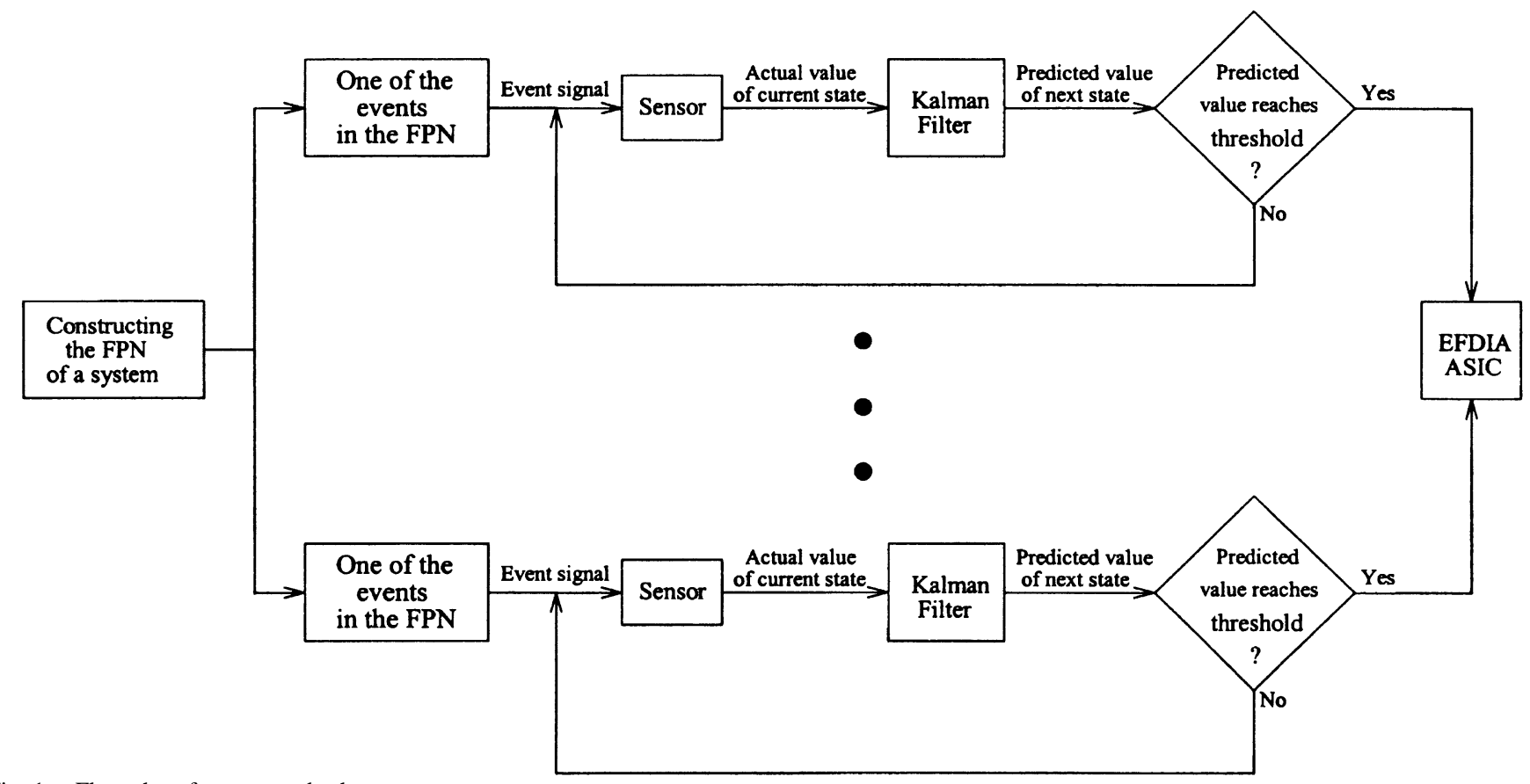

Fig. 1. Flow chart for proposed scheme.

\section{INTRODUCTION}

H IGH-QUALITY and excellent-performance of a system are always goals which engineers strive to achieve. Reliability engineering integrates quality and performance from the beginning to the end of a system life [1]. Therefore, reliability can be treated as the time-dimensional quality of a system. Reliability is affected by every stage throughout the system life, including its development, design, production, quality control, shipping, installation, operation, and maintenance. Consequently, paying attention to each of the stages promotes reliability. Specifically, in the onsite operation phase, failures are the main causes of worsened performance and degraded reliability. Accordingly, failure avoidance is the main approach to reliability assurance. There are 3 main types of maintenance: IM, PM, and CM [2]. The purpose of IM is to reduce or eliminate-entirely the need for maintenance: IM is performed at the design phase of a system and emphasizes elimination of failures. There are many restrictions for a designer, however, such as space, budget, and market requirements. Usually product reliability is related to the product price. On the other hand, CM is the repair performed after failure occurs. PM means all actions intended to keep equipment in good operating condition and to avoid failures [2]. PM should be able to indicate when a failure is about to occur, so that repair can be performed before such failure causes damage or capital-investment loss. Hence, PM is an effective approach to promoting reliability [3]. Time-based and condition-based maintenance are 2 major approaches for PM. In contrast, condition-based maintenance can be a better and more cost-effective type of maintenance than time-based maintenance [4]. Irrespective of the approach adopted for PM, the key point is whether a failure can be detected early or even predicted. If the predicted future state-variables indicate a device is going to fail, then the failure can be prevented in time by PM. Nevertheless, future-state variables should be accurately predicted at a reasonably long time ahead of failure occurrence [5], [6].

Many methods have been proposed for failure prediction such as:

- statistical knowledge of the reliability parameters [7], [8];

- neural network studies [9];

- understanding the failure mechanism of damaged products [10].

This paper proposes a condition-based failure prediction and processing scheme for PM. Fig. 1 shows the flow chart for this scheme.

First, a Petri net dealing with system failure, FPN, must be established, either transformed from a system fault tree or constructed directly [4]. Each event in the FPN is continuously monitored by an adequate sensor and the information is fed to a Kalman filter. Actual values of the event acquired by the monitor sensor are fed into the corresponding Kalman filter to execute state estimation. Based upon the current state, the Kalman filter provides a predicted value of the next state for the corresponding event at every time interval $T$. Each event has a prescribed failure threshold, and the predicted value is compared with that prescribed failure threshold to judge whether the monitored event has failed after $T$ or is still within the established threshold. Once the estimated value reaches the threshold, the failure is predicted. Accordingly, the current state is a warning state and the PM needs to be performed. Subsequently, the EFDIA ASIC [11] is activated to process this warning situation, which includes an alarm, fault isolation, system state description, regulation or auto shutdown, and progress recording.

\section{Control Chart AND Threshold}

A failure threshold is a value used to judge if an equipment failure occurs or not. It is prescribed as the measurement value 


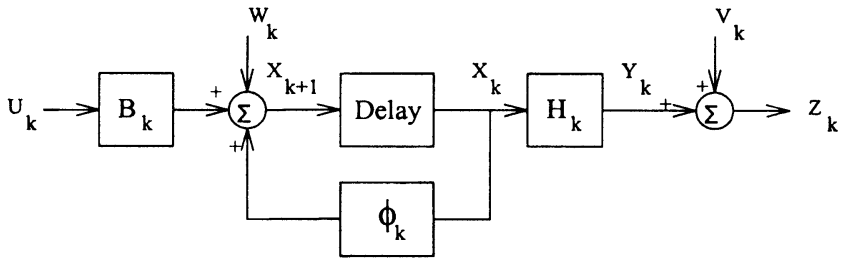

Fig. 2. Block diagram of a discrete system.

that is taken just prior to, or at the time of, failure. Life testing is one method to obtain such data, and can be performed by field engineers or users. Usually, the mean value of a failure-probability-function that is established from tests of manufacturers is a theoretical value for the threshold.

Once the threshold has been determined, a margin of safety should be added to account for variations in early failure detection. The safety margin can be determined by the requirement of lead-time for PM or evaluation of the physical properties and actual operating conditions of various systems. The lower the warning value is set, the greater is the assurance that PM will be done prior to failure [2]; but this also means more labor manpower and cost will be expended. Theoretically, triple the standard deviation is one possible choice in prescribing a warning value [3]. On the basis of failure thresholds and warning values, a control chart can be constructed to conduct limit-control.

Failures can be detected by comparing actual with nominal quantities, and fault isolation can be detected by comparing actual with fault quantities [12]. Consequently, an instrumentation system should be set up for PM, to acquire actual quantities at measurement points. In addition to being used for comparison, acquired quantities can be stored to establish a database for modifying predetermined failure thresholds and warning values. The performance of some systems depends on external conditions. For example, the output current of a power generator varies with the load, which changes with time during the day. Another example is the flow rate of a river, which is different in the 4 seasons. Hence, thresholds and warning-values can be varied according to a scheduled scheme that accomplishes adaptive adjustment for those values. The situation is called 'error' in this paper whenever the acquired quantity exceeds the prescribed low (high) warning value but falls within the low (high) warning value and low (high) failure threshold.

\section{KALMAN FILTERING}

\section{A. System Model}

Fig. 2 shows the block diagram of a discrete system. The state equations [13] are:

$$
\begin{aligned}
X_{k+1} & =\phi_{k} \cdot X_{k}+B_{k} \cdot U_{k}+W_{k} ; \\
Y_{k} & =H_{k} \cdot X_{k} ; \\
Z_{k} & =Y_{k}+V_{k} .
\end{aligned}
$$

Substituting (1) into (2) yields:

$$
Z_{k}=H_{k} \cdot X_{k}+V_{k}
$$

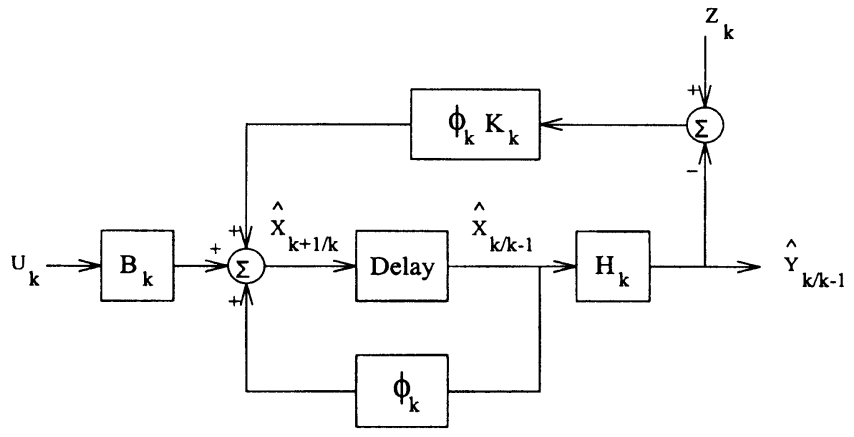

Fig. 3. Block diagram of Kalman filter.

The covariance matrices for $W_{k}$ and $V_{k}$ are:

$$
\begin{aligned}
E\left[W_{k} \cdot W_{i}^{T}\right] & = \begin{cases}Q_{k} & i=k \\
0 & i \neq k ;\end{cases} \\
E\left[V_{k} \cdot V_{i}^{T}\right] & = \begin{cases}R_{k} & i=k \\
0 & i \neq k\end{cases} \\
E\left[W_{k} \cdot V_{i}^{T}\right] & =0, \text { for all } k, i
\end{aligned}
$$

It follows that both $Q_{k}$ and $R_{k}$ are symmetric and positive definite [14].

\section{B. State Estimation}

State estimation aims to guess the value of $X_{k}$ by using measured data: $Z_{0}, Z_{1}, \ldots, Z_{k-1} . \hat{\mathbf{X}}_{k / k-1}$ is the prior estimate of $\mathbf{X}$, and $\hat{\mathbf{X}}_{k / k}$ is the posterior estimate of $X$ [14]. The estimation problem begins with: no prior measurements. Thus, the stochastic portion of the initial estimate is zero if the stochastic process-mean is zero: $\mathbf{X}_{0 /-1}$ is only driven by the initial states resulting from deterministic input.

The Kalman filter is a copy of the original system and is driven by the estimation error and the deterministic input. Fig. 3 shows the block diagram of the filter structure. The filter is used to improve the prior estimate, using $Z_{k}$ to yield the posterior estimate. Fig. 3 shows that the 1-step-ahead estimate is formulated as [14]:

$$
\begin{aligned}
\hat{\mathbf{X}}_{k+1 / k}= & \phi_{k} \cdot \hat{\mathbf{X}}_{k / k-1}+\phi_{k} \cdot K_{k} \cdot\left(Z_{k}-H_{k} \cdot \hat{\mathbf{X}}_{k / k-1}\right) \\
& +B_{k} \cdot U_{k} \\
= & \phi_{k}\left[\hat{\mathbf{X}}_{k / k-1}+K_{k} \cdot\left(Z_{k}-H_{k} \hat{\mathbf{X}}_{k / k-1}\right)\right] \\
& +B_{k} \cdot U_{k} \\
= & \phi_{k} \hat{\mathbf{X}}_{k / k}+B_{k} \cdot U_{k} .
\end{aligned}
$$

According to the properties of the Kalman filter, several remarks on Kalman estimation are:

1) Because $K_{k}$ is optimal, the posterior estimate $\hat{\mathbf{X}}_{k / k}$ is an optimal estimate.

2) Fig. 4 summarizes the recursive steps for constructing a 1 -step estimator.

3) The recursive loop has 2 kinds of updating: a) Deriving $\hat{\mathbf{X}}_{k / k}$ and $P_{k / k}$ from $\hat{\mathbf{X}}_{k / k-1}$ and $P_{k / k-1}$ are measurement-updates; b) Projecting $\hat{\mathbf{X}}_{k / k}$ and $P_{k / k}$ to $\hat{\mathbf{X}}_{k+1 / k}$ and $P_{k+1 / k}$ are time-updates. 


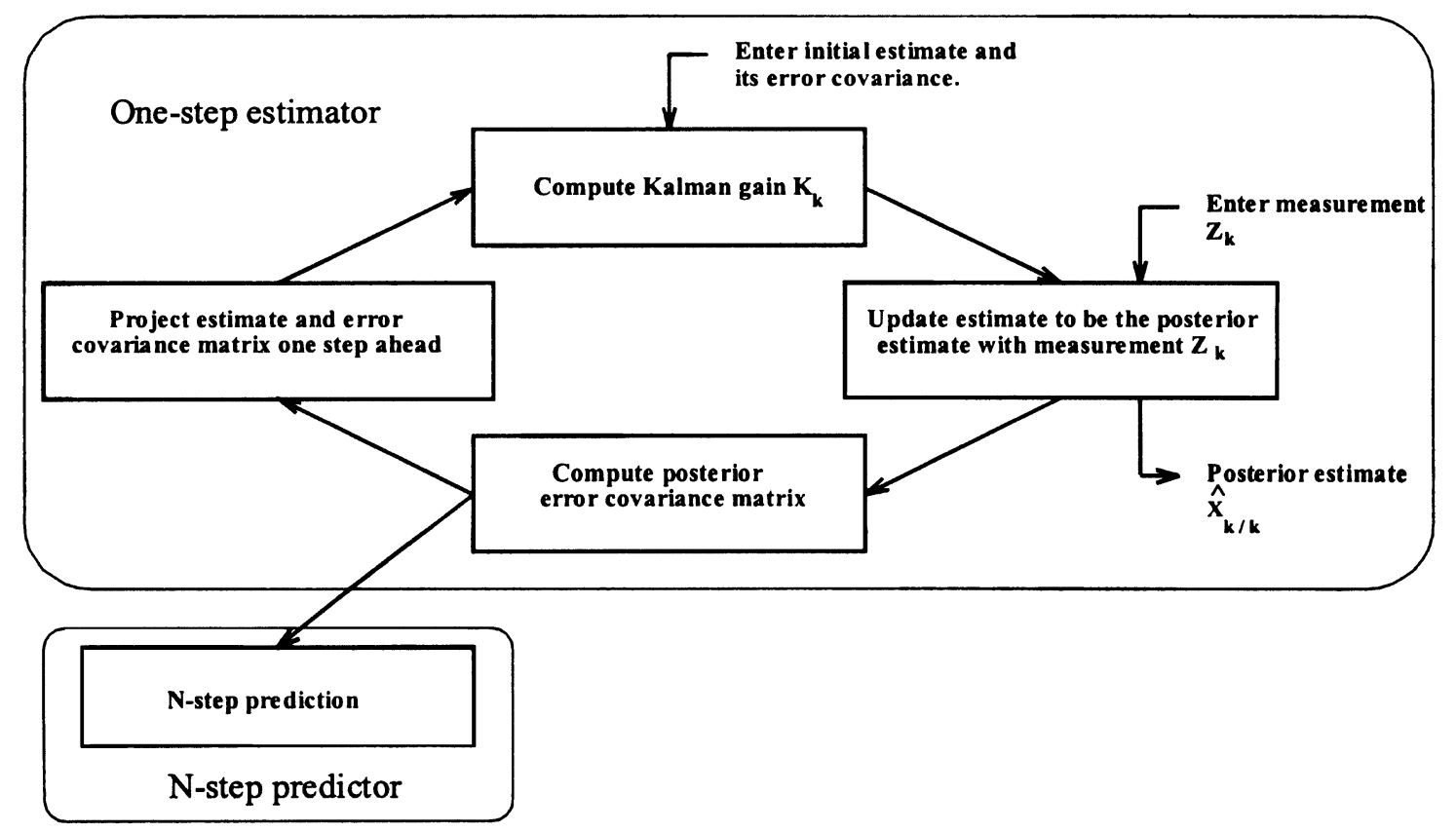

Fig. 4. 1-Step estimator and $N$-step predictor.

4) Initial conditions $\left.\left(\hat{\mathbf{X}}_{0 /-1}\right), P_{0 /-1}, \phi_{0}, H_{0}, Q_{0}, R_{0}\right)$ have to be known to start recursive steps.

5) The increment time for every step in Kalman prediction, $T$, should be set at a reasonably long time ahead of failure occurrence for performing PM. However, the shorter the $T$ used, the higher the prediction accuracy it achieves [15].

\section{Prediction}

The estimate resulting from recursive steps in Fig. 4 is a 1 -step-ahead prediction. Based on the posterior estimate, the state that is $N$ steps ahead of the $Z_{k}$ can be predicted by using the ARMA model [14]. Equations for $N$-step-ahead prediction $(N \geq 2)$ are derived as in [15]:

$$
\begin{aligned}
\hat{\mathbf{X}}_{k+N / k}= & \left(\prod_{i=k+N-1}^{k} \phi_{i}\right) \cdot \hat{\mathbf{X}}_{k / k} \\
& +\sum_{m=k}^{k+N-2}\left[\left(\prod_{i=k+N-1}^{m+1} \phi_{i}\right) \cdot B_{m} \cdot U_{m}\right] \\
& +B_{k+N-1} \cdot U_{k+N-1} ; \\
P_{k+N / k}= & \left(\prod_{i=k+N-1}^{k} \phi_{i}\right) \cdot P_{k / k} \cdot\left(\prod_{j=k}^{k+N-1} \phi_{j}^{T}\right) \\
& +\sum_{m=k}^{k+N-2}\left[\left(\prod_{i=k+N-1}^{m+1} \phi_{i}\right)\right. \\
& \left.\cdot Q_{m} \cdot\left(\prod_{j=m+1}^{k+N-1} \phi_{j}^{T}\right)\right]+Q_{k+N-1} .
\end{aligned}
$$

The $N$-step predictor is an appendage of the 1-step estimation loop [14]; it is also shown in Fig. 4. Because the current pre- dicted value is assumed to be the initial value for the next prediction, the more steps the predictor predicts, the larger the error [15].

The failure prediction simulation on a DC motor for preventive maintenance by state estimation using the Kalman filter was reported in [15]. Failure times were generated by Monte Carlo simulation and predicted by the Kalman filter. 1-step-ahead and 2 -step-ahead predictions are conducted. The resulting prediction errors are sufficiently small in both predictions. An experiment of state estimation for predictive maintenance using the Kalman filter on a DC motor has also been performed. [16] shows that the resultant prediction errors for 1-step-ahead prediction are acceptable; and the shorter the increment time for every step in Kalman prediction uses, the higher prediction accuracy it achieves.

\section{Petri Nets AND EFDiA}

\section{A. Introduction}

A Petri net is a general-purpose graphic tool to describe relations existing between conditions and events [17]. The basic symbols of Petri nets include [18]:

$\bigcirc$ : Place, drawn as a circle, denotes an event;

-: Immediate transition, drawn as a thin bar, denotes an event transfer with no delay time;

-: Timed transition, drawn as a thick bar, denotes event transfer with a period of delay time;

/* Note to compositor: the - is intended to be about 1/16 inch thick. I do not know how to do it. Please make it happen in the final manuscript. */

$\uparrow$ : Arc, drawn as an arrow, between places and transitions;

-: Token, drawn as a dot, contained in places, denotes the data;

: Inhibitor arc, drawn as a line with a circle end, between
places and transitions. 


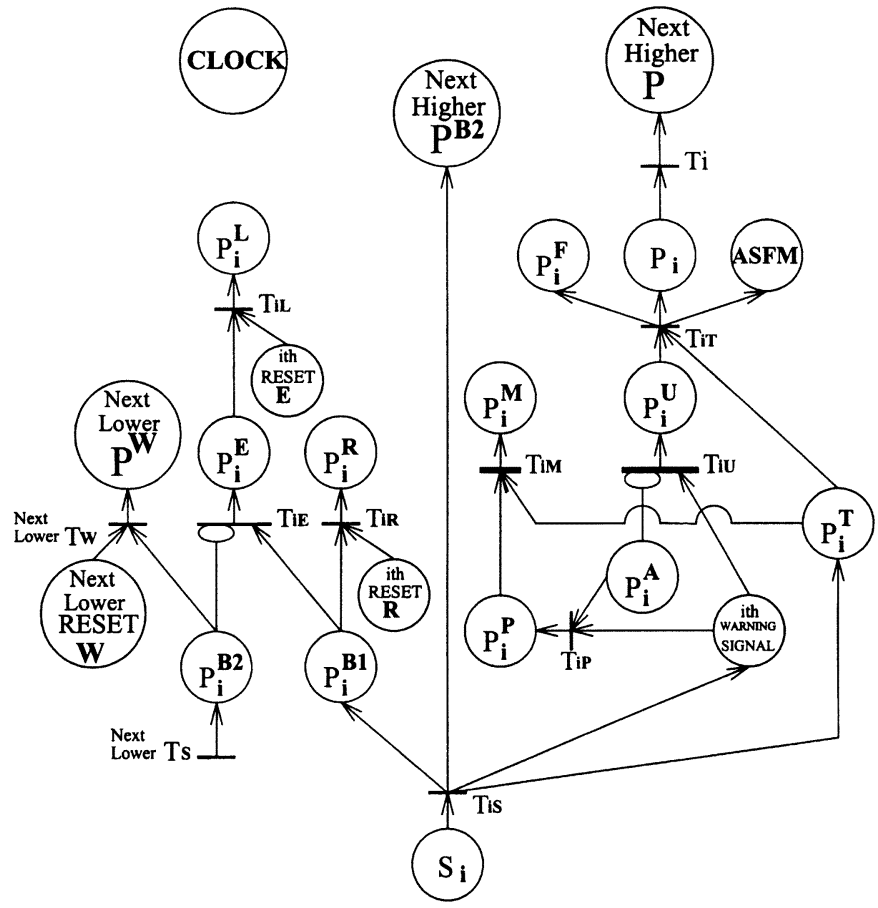

Fig. 5. Early failure detection and isolation arrangement (EFDIA).

Places contain dots, the representation of tokens, being the specific marking of a Petri net [19]. The transition is said to fire, if input places satisfy an enabling condition. Transition firing removes 1 token from all of its input places and puts 1 token into all of its output places [20]. There are 3 types of transitions that are time-based classified [17]. Transitions with no time delay are 'immediate transitions', while those that need a certain constant period of time for transition are 'timed transitions'. The third type is a 'stochastic transition' and is used for modeling a process with random time. Hence the Petri net is a powerful tool for modeling various systems.

\section{B. The EFDIA}

An EFDIA [4] is used in this paper. It is a hybrid Petri net that includes three kinds of Petri sub-nets: ordinary, inhibitor-arc type, and timed. In an FPN, for PM optimization, each place with a monitor sensor is equipped with an EFDIA that facilitates alarm, early failure detection, fault isolation, event count, system state description, and automatic shutdown or regulation. In this context, a cause-consequence type of FPN is drawn in fault-free style with basic events at the bottom and the final undesirable event at the top (see Fig. 10). Fig. 5 shows EFDIA; the symbols are defined in the following list.

1) $n$ : total number of sensing points in a FPN.

2) $i$ : sequence number, $1 \leq i \leq n$.

3) $M(P)_{k}$ : marking of place $P$ at time $k \cdot T$, representing the token quantity of place $P$ at time $k \cdot T$, $k=1,2,3, \ldots$.

4) $P_{i}$ : place $\# i$ of FPN, $M\left(P_{i}\right)=1$ if the failure represented by $P_{i}$ occurs.

5) $T_{i}$ : transition $\# i$ of FPN, representing the time duration.

6) $S_{i}$ : a place for the Kalman-predicted indicator value of the sensing signal of $P_{i}$ Place. $S_{i}$ generates a token such that $M\left(S_{i}\right)=1$ if the next state of $S_{i}$ that is predicted by the Kalman filter reaches the prescribed threshold.

7) $T_{i, E}$ : error transition of $P_{i}$; an immediate transition.

8) $T_{i, L}$ : error times $\log$ transition of $P_{i}$; an immediate transition.

9) $T_{i, M}$ : maintained transition, representing the transitional time from when the PM action for $P_{i}$ is taken to when $P_{i}$ is maintained; a timed transition.

10) $T_{i, P}$ : processing transition of $P_{i}$; an immediate transition.

11) $T_{i, R}$ : reset transition of $P_{i}$; an immediate transition.

12) $T_{i, S}$ : sensing transition of $P_{i}$; an immediate transition.

13) $T_{i, T}$ : transfer transition of $P_{i}$; an immediate transition.

14) $T_{i, U}$ : unprocessed transition of $P_{i}$, representing the transitional time from when warning-signal \# $i$ appears, to when $P_{i}$ failure occurs, a timed transition.

15) Next Lower $T_{W}$ : warning times $\log$ transition of the corresponding next lower level $P^{W}$; an immediate transition; the number of the Next Lower $T_{W}$ should equal the number of inhibitor arcs of transition $T_{i, E}$.

16) $P_{i}^{A}$ : PM action taken place of $P_{i} ; P_{i}^{A}$ generates a token such that $M\left(P_{i}^{A}\right)=1$ if the PM action for $P_{i}$ is taken.

17) $P_{i}^{B, j}$ : buffer-place $\# j$ of $P_{i}$; for tokens to stay temporarily, $j=1$ to $x ; x$ is the number of input arcs for $P_{i} . P_{i}^{B, j}$ is unnecessary when $P_{i}$ is a basic place in a FPN. A basic place is a place where there is no place lower than it in a Petri net.

18) $P_{i}^{E}$ : error indication place of $P_{i} ; M\left(P_{i}^{E}\right)=1$ after $T_{i, E}$ fires if the $M\left(S_{i}\right)=1$ situation is generated by $P_{i}$ itself, but not aroused by lower-level places (for fault isolation).

19) $P_{i}^{F}$ : failure counter place of $P_{i} ; M\left(P_{i}^{F}\right)$ represents failure times $\log$ number of $P_{i} ; M\left(P_{i}^{F}\right)$ increases by 1 when $P_{i}$ failure occurs.

20) $P_{i}^{L}$ : error counter place of $P_{i} ; M\left(P_{i}^{L}\right)$ represents the error times $\log$ number of $P_{i} ; M\left(P_{i}^{L}\right)$ increases by 1 when $P_{i}$ error occurs.

21) $P_{i}^{M}$ : maintenance counter place of $P_{i} ; M\left(P_{i}^{M}\right)$ represents maintenance times log number of $P_{i} ; M\left(P_{i}^{M}\right)$ increases by 1 when the $M\left(S_{i}\right)=1$ situation is maintained.

22) $P_{i}^{P}$ : processing place of $P_{i}$, representing $P_{i}$ in a beingmaintained situation.

23) $P_{i}^{R}$ : reset counter place of $P_{i} ; M\left(P_{i}^{R}\right)$ represents the warning times $\log$ number of $P_{i}$ that are aroused by lower-level places: the reset times of the RESET R \# $i$.

24) $P_{i}^{T}$ : transitional place of $P_{i}$, representing a transitional state inserted between $S_{i}$ and $P_{i}$, which is the original path from $S_{i}$ to $P_{i}$ without EFDIA constructed.

25) $P_{i}^{U}$ : unprocessed place of $P_{i}$, representing: the error of $P_{i}$ not corrected.

26) Next Lower $P^{W}$ : warning counter place of the next lower $P ; M$ (Next Lower $P^{W}$ ) represents the warning times $\log$ number of the next lower $P$ no matter from where warning cause arises. The number of the Next Lower $P^{W}$ should equal the number of inhibitor arcs of transition, $T_{i, E}$.

27) RESET E \# $i$ : reset $\mathrm{E}$ place of $P_{i}$, representing a reset signal for $P_{i}^{E}$, generates a token once it is triggered. 


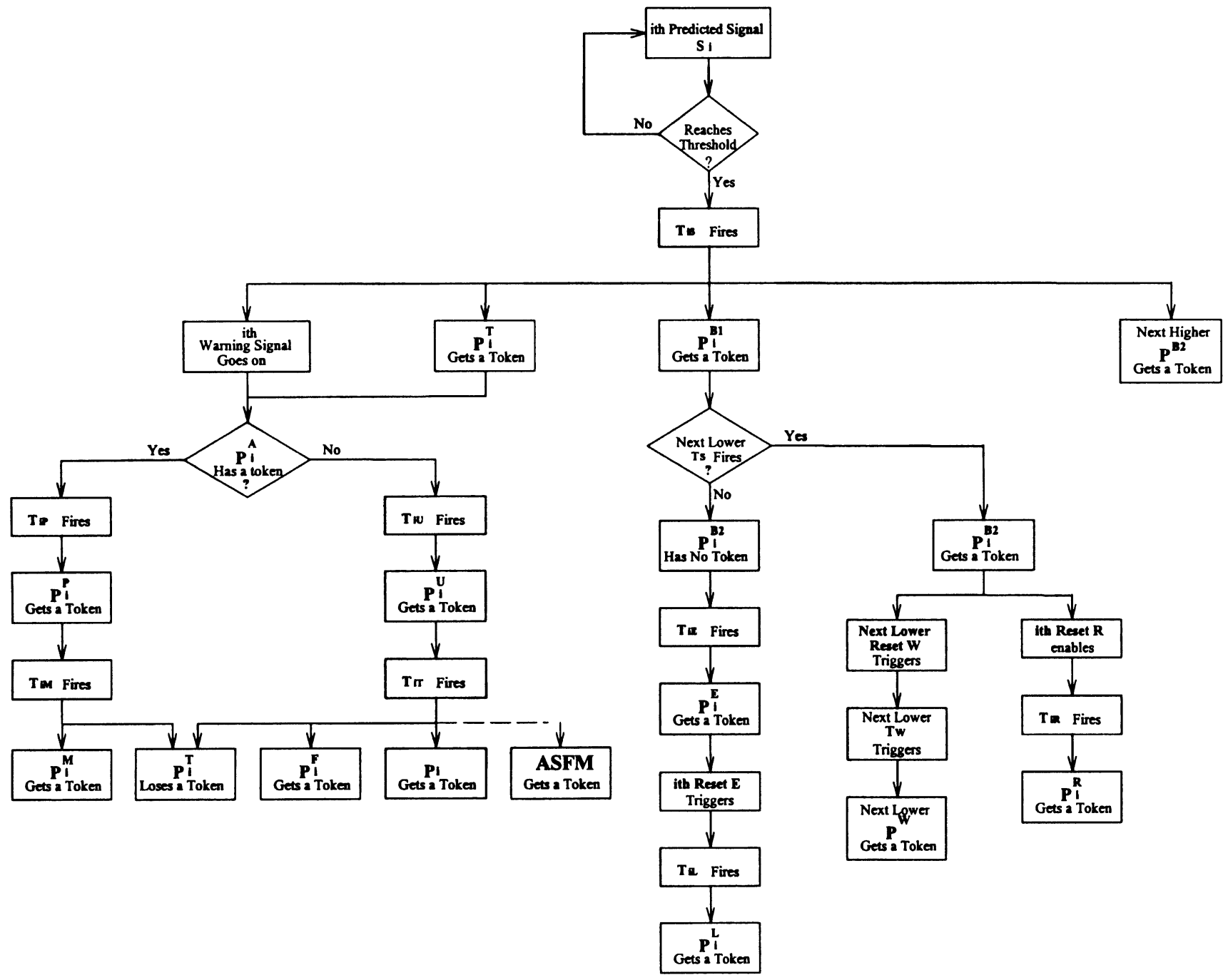

Fig. 6. Flowchart of the EFDIA.

28) RESET $\mathrm{R} \# i$ : reset $\mathrm{R}$ place of $P_{i}$, representing a reset signal for $P_{i}^{B 1}$, generates a token when it is triggered.

29) Next Lower RESET W: reset $W$ place of the corresponding next lower level $P^{W}$; representing a reset signal for $P_{i}^{B, j}$ ( $j$ is the sequence number of the next lower place); generates a token once it is triggered; the number of the Next Lower RESET W should equal number of inhibitor arcs of transition $T_{i, E}$.

30) ASFM: Automatic Shutdown or Feedback Mechanism place; e.g., an air-conditioning or ventilation system is a feedback mechanism for an over temperature module.

31) WARNING SIGNAL $\# i$ : warning signal place for $P_{i}$.

32) Clock: a clock is embedded to indicate and record the time of the occurrence for each event.

Conventionally, a flowchart is an easy visual representation for understanding operational steps. Therefore, the operational steps for EFDIA are summarized in a flowchart (Fig. 6).

\section{Properties/Capabilities of EFDIA}

1) Alarm. EFDIA provides alarm capability whenever a predicted over-threshold situation occurs, by triggering WARNING SIGNAL \# $i$ for the associated place.
2) Early failure detection. EFDIA is capable of early failure detection, because the alarm function operates whenever the Kalman-predicted future-state variable reaches the corresponding prescribed failure threshold. Thus the abnormal situation is detected before failure occurs. The lead-time of early detection can be obtained by extrapolating the curve in a control chart with a line slope that is constructed by the latest 2 sampled points on the curve [21]. The lead-time is the period between the 'time point where the warning value is exceeded' and the 'intersection of the extended line and the time axis'. The lead-time obtained from the control chart for the 'monitored channel to be predicted' is one possible value for the increment time for every step in the Kalman prediction.

3) Fault isolation. The cause(s) of malfunction of a system can be anywhere within the system. However, because malfunction causes are constrained by the logic relations of the FPN, they can be isolated by the $T_{i, E}$ via the indication of the $P_{i}^{E}$. The error is located at place \#i if $M\left(P_{i}^{E}\right)=1$. Otherwise, the error of place $\# i$ arises from the lower-level place(s) even if warning signal \#i appears. 


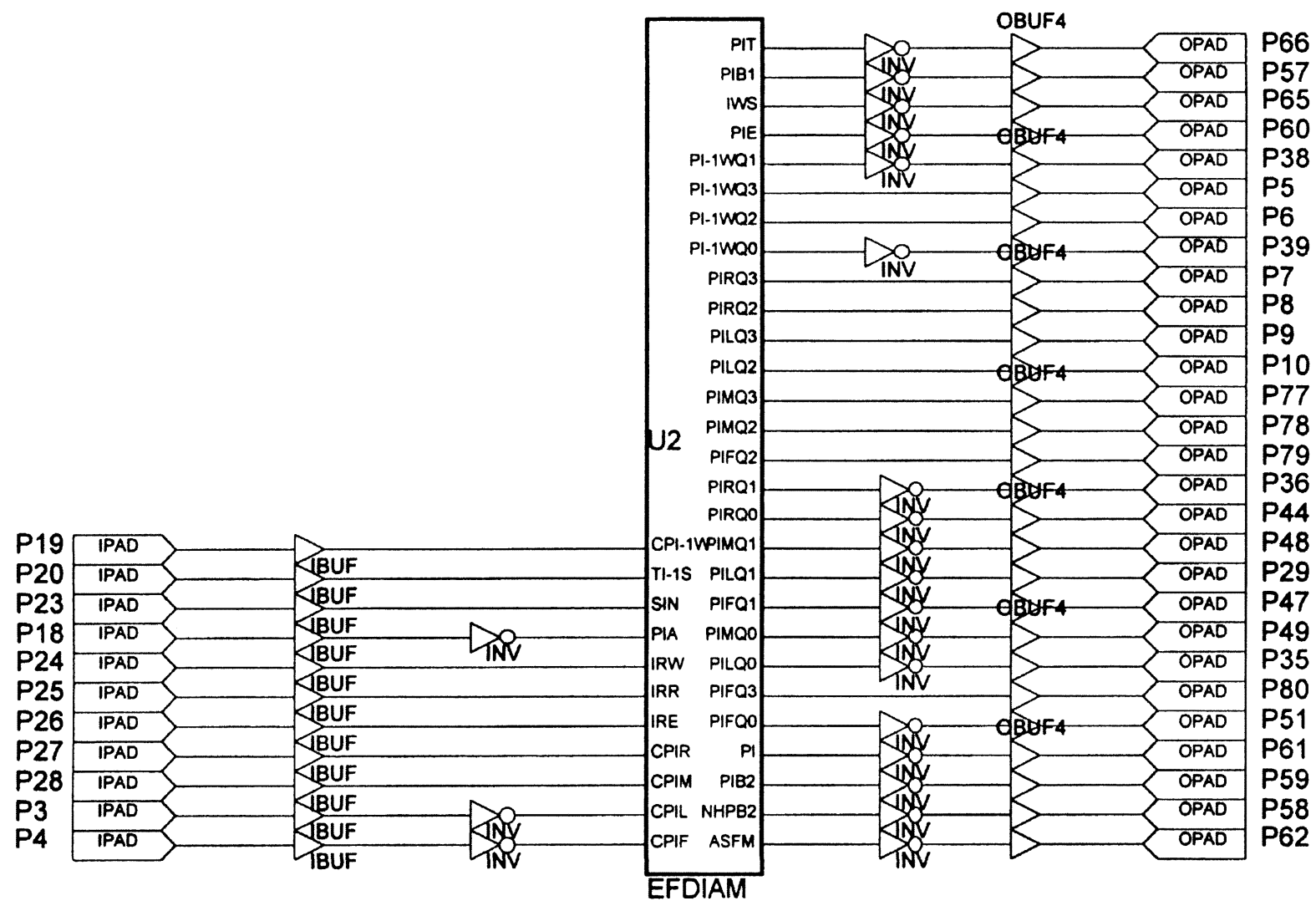

Fig. 7. EFDIA macro symbol.

4) Event count. All the counters designated in EFDIA, record the associated occurrence multiplicities of events. By incorporating a time clock, the associated rates can be obtained at the same time. The following items can be derived from EFDIA:

a) Failure rate of place $\# i: M\left(P_{i}^{F}\right) / t$;

b) Error rate of place $\# i: M\left(P_{i}^{L}\right) / t$;

c) Maintenance rate of place $\# i: M\left(P_{i}^{M}\right) / t$;

d) Alarm rate of place $\# i: M\left(P_{i}^{W}\right) / t$.

From these rates, two advantages can be obtained:

a) If subsystem $\# i$ is maintained whenever a failure is predicted, the failure rate of place \# $i$ can be minimized such that the system reliability is improved.

b) All the rates can be recorded as historical data so as to perform statistical prediction of system failure (by failure rate and error rate), and to be able to derive the time needed for maintenance (by maintenance rate) of each subsystem.

5) System state description. The system state is clearly visible by the indication of every place in EFDIA. The following parameters are defined to account for system state:

a) $\mathbf{M}_{k}=\left[M\left(P_{1}\right), M\left(P_{2}\right), \ldots, M\left(P_{n}\right)\right]^{T}$ : marking of the FPN at state $k$;

b) $\mathbf{S}_{k}=\left[M\left(S_{1}\right), M\left(S_{2}\right), \ldots, M\left(S_{n}\right)\right]^{T}$ : predicted sensing signal matrix at state $k$;

c) $\mathbf{L}_{k}=\left[M\left(P_{1}^{M}\right), M\left(P_{2}^{M}\right), \ldots, M\left(P_{n}^{M}\right)\right]^{T}$ : maintenance $\log$ matrix at state $k$; d) $\mathbf{F}_{k}=\left[M\left(P_{1}^{F}\right), M\left(P_{2}^{F}\right), \ldots, M\left(P_{n}^{F}\right)\right]^{T}$ : failure number $\log$ matrix at state $k$;

e) $\mathbf{E}_{k}=\left[M\left(P_{1}^{E}\right), M\left(P_{2}^{E}\right), \ldots, M\left(P_{n}^{E}\right)\right]^{T}$ : error indication matrix at state $k$; entry \# $i$ indicates that the error is located at the place \# $i$ if the value of entry $\# i$ is 1 .

6) Automatic shutdown or regulation. Automatic shutdown or regulation capability can be provided by EFDIA through triggering the ASFM place. ASFM is intended to prevent a higher-level fault or system breakdown by automatic shutdown or regulation. It should be incorporated into the places that can cause a safety problem in a FPN.

7) Time recording. The time at which each event occurs can be recorded by the embedded CLOCK. This is required for failure analysis.

\section{IMPLEMENTATION OF EFDIA}

A system can be modeled into a Petri-net to express not only static behaviors such as logical relations between components of the system, but also dynamic behaviors like operating sequence or failure occurrence of the system. Because Petri nets are state machines [22], it is feasible to make Petri nets to perform those capabilities. Hardware implementation of Petri nets actualizes state machines that are converted from Petri nets to logic circuits. Nowadays, IC are becoming not only smaller and more powerful but also faster and cheaper. As a result, ASIC are widely used. In practice, Petri nets can be implemented as ASIC, to perform specific functions without user intervention. 


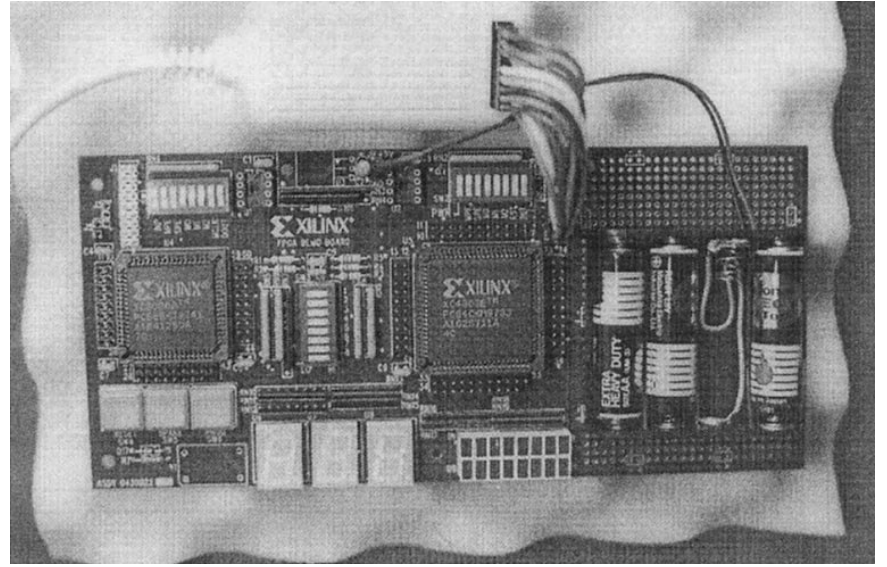

Fig. 8. The down-loaded Demonstration Board.

Mainly because of the programmable capability, FPGA are suitable for hardware implementation of Petri nets. This study uses a Xilinx FPGA [23] as the design tool to implement Petri nets.

The EFDIA circuit can be integrated into a 39-pin ASIC. Fig. 7 shows the macro symbol for EFDIA. Hence, the EFDIA Petri net can become an ASIC after downloading this EFDIA macro to a Xilinx FPGA board [24]. The correspondence between EFDIA pin names (Fig. 7) and EFDIA Petri net symbol names (Fig. 5) are:

1) Input pins

a) CPI-1W: clear signal (implicit in Fig. 5) for Next Lower $P^{W}$ counter;

b) TI-1S: Next Lower $T_{S}$;

c) SIN: $S_{i}$

d) PIA: $\# P_{i}^{A}$;

e) IRW: Next Lower Reset $W$;

f) IRR: Reset $\mathrm{R} \# i$;

g) IRE: Reset E \# $i$;

h) CPIR: clear signal, implicit in Fig. 5, for $P_{i}^{R}$ counter;

i) CPIM: clear signal, implicit in Fig. 5, for $P_{i}^{M}$ counter;

j) CPIL: clear signal, implicit in Fig. 5, for $P_{i}^{L}$ counter;

k) CPIF: clear signal, implicit in Fig. 5, for $P_{i}^{F}$ counter;

2) Output pins
a) PIT: $P_{i}^{T}$;
b) PIB1: $P_{i}^{B 1}$;
c) IWS: WARNING SIGNAL \# $i$;
d) PIE: $P_{i}^{E}$;
e) PI-1WQ0 $\sim$ PI-1WQ3: Next Lower $P^{W}$ counter;
f) PIRQ0 $\sim$ PIRQ3: $P_{i}^{R}$ counter;
g) PILQ0 $\sim$ PILQ3: $P_{i}^{L}$ counter;
h) PIFQ0 $\sim$ PIFQ3: $P_{i}^{F}$ counter;
i) PIMQ0 $\sim$ PIMQ3: $P_{i}^{M}$ counter;
j) PI: $P_{i}$;
k) PIB2: $P_{i}^{B 2}$;
1) NHPB2: Next Higher $P^{B 2}$;
m) ASFM: ASFM.

Fig. 8 shows the down-loaded Demonstration Board [11].

\section{EXAMPLE}

A thermal power plant is an example for early failure detection and processing by using the scheme in this paper. Fig. 9 is the system block diagram for the thermal power plant. To construct the FPN, 8 sensors are selected to be installed at the associated test points to acquire data. Fig. 9 shows sensor types, locations, and associated sensing signals. Fig. 10 shows the resultant FPN of this system.

Fig. 11 shows the FPN for the thermal power plant with EFDIA. It is a result of appending an EFDIA to each place with a monitor sensor in Fig. 10: $P_{1}$ to $P_{8}$. The logic relations among all places in Fig. 10 are retained in Fig. 11 (the shadowed portion). At basic places of the FPN $\left(P_{1}, P_{4}, P_{5}\right)$, the function for testing whether the error cause is from the "next lower place' or not is unnecessary. The following 2 situations are used to demonstrate the function of EFDIA in this system.

1) Let the Kalman-predicted value of the monitored signal for fuel flow $\left(S_{1}\right)$ in Fig. 11) reach the prescribed threshold. Subsequently, $T_{1 S}$ fires such that the 1 st WARNING SIGNAL is produced and each of $P_{1}^{T}, P_{2}^{B 2}$ and $P_{1}^{E}$ obtains a token. $M\left(P_{1}^{T}\right)=1$ represents the fuel flow rate that is at an error situation, and is a transitional state between normal and faulty. There is a lead-time from then until the $P_{1}$ failure really happens. If the PM action takes place during the lead-time, then $P_{1}^{A}$ generates a token such that $T_{1 P}$ fires so that the token in $P_{1}^{A}$ together with the token in the 1st WARNING SIGNAL move to $P_{1}^{P}$. The subsystem is being maintained and the 1st WARNING SIGNAL goes off at this moment. $T_{1 M}$ fires if the PM action is finished. Subsequently, the tokens in $P_{1}^{P}$ and $P_{1}^{T}$ move to $P_{1}^{M}$ : this error has been corrected. The marking of $P_{1}^{M}$ (the maintenance-times $\log$ number for $P_{1}$ ) increases by 1 . On the other hand, if the PM action does not take place soon enough, then $T_{1 U}$ fires such that $P_{1}^{U}$ obtains a token. Consequently, the tokens in $P_{1}^{U}$ and $P_{1}^{T}$ move to $P_{1}$. Hence, $P_{1}$ failure occurs. At the same time, $P_{1}^{F}$ obtains a token: the failure-times $\log$ number for $P_{1}$ increases by 1 . Because of the logic relation between $P_{1}$ and $P_{2}$, the Kalman-predicted value of the monitored signal for $P_{2}\left(S_{2}\right)$ exceeds the prescribed threshold due to $P_{1}$ failure. Accordingly, the 2nd WARNING SIGNAL is produced and each of $P_{2}^{T}, P_{3}^{B 2}, P_{2}^{B 1}$, obtains a token. $T_{2 E}$ is inhibited by the token in $P_{2}^{B 2}$, such that the tokens in $P_{2}^{B 1}$ and $P_{2}^{B 2}$ move to $P_{2}^{R}$ and $P_{1}^{W}$ after triggering the 2nd RESET R and the 1st RESET $\mathrm{W}$, respectively. Hence this error is located at $P_{1}$, whereas $M\left(P_{2}^{L}\right)$ does not increase.

2) Let the Kalman-predicted value of the monitored signal for shaft rotation speed $\left(S_{7}\right.$ in Fig. 11) exceeds the prescribed failure threshold spontaneously while all $S_{3}, S_{4}$, and $S_{6}$ are at the usual condition. As a result, $T_{7 S}$ fires such that the 7th WARNING SIGNAL goes on. Simultaneously, each of $P_{7}^{T}, P_{7}^{B 1}$, and $P_{8}^{B 2}$ obtains a token. Similarly, as $S_{1}$ exceeds the prescribed threshold, then $M\left(P_{7}^{M}\right)$ increases by 1 if the PM action for $P_{7}$ takes place in time. Otherwise, the $P_{7}$ failure occurs such that 


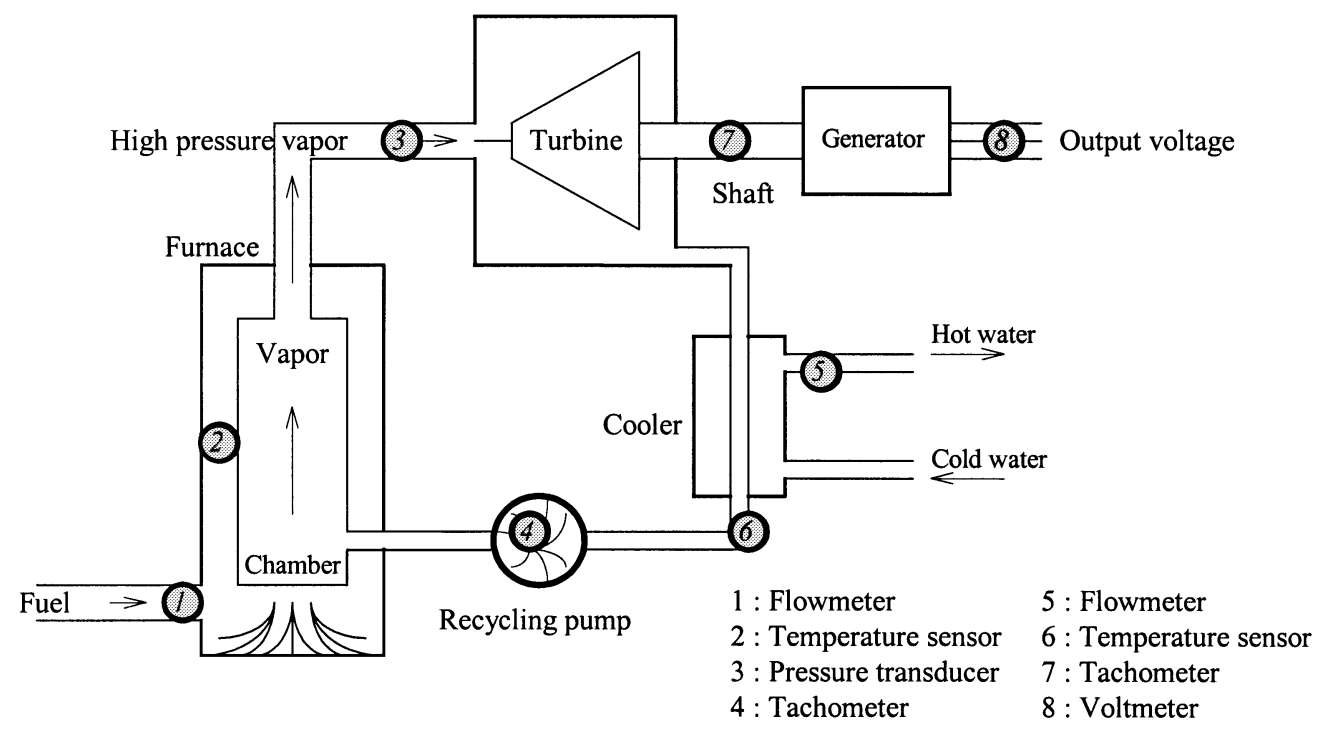

Fig. 9. System block-diagram of a thermal power plant.

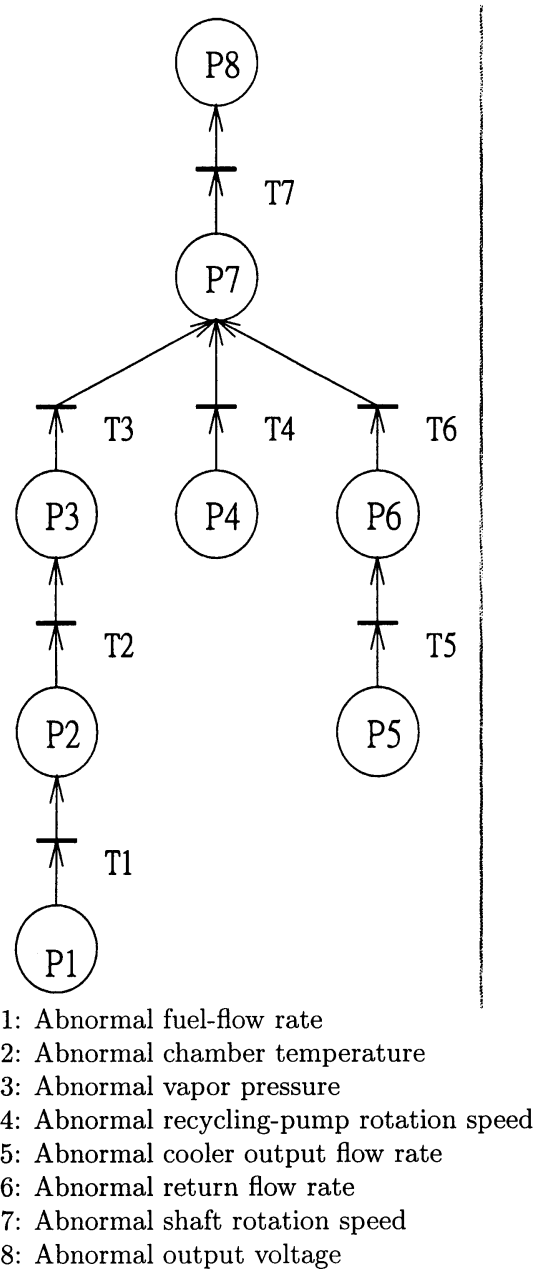

Fig. 10. The FPN for the thermal power plant.

$M\left(P_{7}^{F}\right)$ increases by 1 . However, because $P_{7}^{B 2}, P_{7}^{B 3}$, and $P_{7}^{B 4}$ are empty, then $T_{7 E}$ fires such that $P_{7}^{E}$ obtains a token. As a result, $M\left(P_{7}^{L}\right)$ (the error times log number for $P_{7}$ ) increases by 1 after triggering the 7th RESET E It indicates that this error is caused by the 7 th monitored signal (the shaft rotation speed), but not by next lower signals (vapor pressure, recycling pump rotation speed, or return flow temperature).

ASFM should be put in the places that can cause safety problems in a FPN. In this example, each of $P_{1}, \ldots, P_{8}$ failures can trigger ASFM.

\section{DISCUSSION}

Knowing when and where a system needs maintenance and economizing capital investment, are 2 of the major problems of maintenance. The scheme in this paper improves the maintenance problem in the following aspects.

1) Before a system failure occurs, the scheme can indicate where and when the failure is going to be.

2) It makes both the health condition and the historical record of maintenance for a system clear at a glance.

3) Scheduled maintenance is enacted, based on a statistical average, which still retains the unavoidable risk that the system might fail before criteria are exceeded: a failure might occur unexpectedly. On the other hand, the actual duty-cycles for a certain part or module might be longer than those averages, thus if they are replaced during scheduled maintenance, that is a waste of the investment. The condition-based scheme avoids these drawbacks.

In contrast to previous papers, this study uses Kalman-filtering instead of parameter-trend to predict the time of failureoccurrence and to determine the PM execution-time. Furthermore, after an upcoming failure is predicted, the followed evolution of the system is processed by an ASIC that is designed by a Petri-net approach. The ASIC has been implemented and tested, which shows the proposed scheme is practical and satisfies the condition-based failure prediction and processing for a thermal power plant and other systems. 


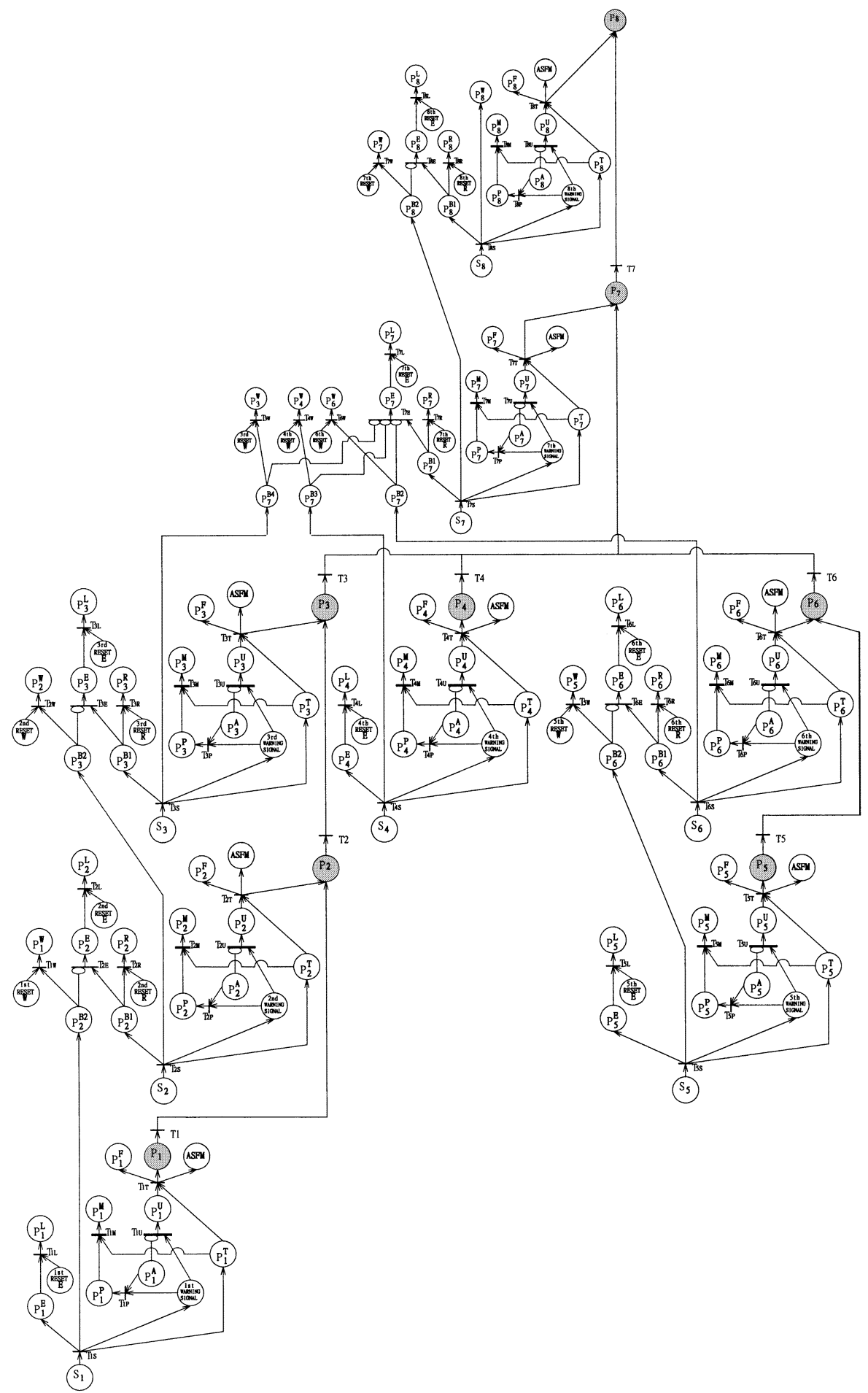

Fig. 11. The FPN for the thermal plant EFDIA.

\section{ACKNOWLEDGMENT}

The author would like to thank the Associate Editor for excellent comments and suggestions that immensely improved the presentation of this paper.

\section{REFERENCES}

[1] E. A. Elsayed, Reliability Engineering: Addison Wesley Longman, 1996.

[2] J. D. Patton, Preventive Maintenance: Instrument Soc. of America, 1983.
[3] S. S. Rao, Reliability-Based Design: McGraw-Hill, 1992.

[4] S. K. Yang and T. S. Liu, "A petri net approach to early failure detection and isolation for preventive maintenance," Qual. Reliab. Eng. Int., vol. 14, pp. 319-330, 1998.

[5] R. Isermann, "Process fault detection based on modeling and estimation methods-A survey," Automatica, vol. 20, no. 4, pp. 387-404, 1984.

[6] A. S. Willsky, "A survey of design methods for failure detection in dynamic systems," Automatica, vol. 12, pp. 601-611, 1976.

[7] O. T. Ogunyemi and P. I. Nelson, "Prediction of gamma failure times," IEEE Trans. Rel., vol. 46, no. 3, pp. 400-405, 1997.

[8] W. Nelson, "Weibull prediction of a future number of failures," Qual. Reliab. Eng. Int., vol. 16, pp. 23-26, 2000. 
[9] E. A. Rietman and M. Beachy, "A study on failure prediction in a plasma reactor," IEEE Trans. Semiconductor Manufacturing, vol. 11, no. 4, pp. 670-680, 1998.

[10] S. N. Kher and G. M. Bubel, "Predicting system-failure risk from unanticipated fiber-breaks in manufacturing," IEEE Trans. Rel., vol. 47, no. 2, pp. 126-130, 1998.

[11] S. K. Yang and T. S. Liu, "Implementation of petri nets using a fieldprogrammable gate array," Qual. Reliab. Eng. Int., vol. 16, pp. 99-116, 2000.

[12] A. Baccigalupi, A. Bernieri, and A. Pietrosanto, "A digital-signal-processor-based measurement system for on-line fault detection," IEEE Trans. Instrumentation and Measurement, vol. 46, no. 3, pp. 731-736, 1997.

[13] B. D. O. Anderson and J. B. Moore, Optimal Filtering: Prentice-Hall, 1979.

[14] R. G. Brown and P. Y. C. Hwang, Introduction to Random Signals and Applied Kalman Filtering: John Wiley \& Sons, 1997, sec. 5.3-5.6, pp. 198-224.

[15] S. K. Yang and T. S. Liu, "State estimation for predictive maintenance using kalman filter," Reliab. Eng. Syst. Saf., vol. 66, pp. 29-39, 1999.

[16] S. K. Yang, "An experiment of state estimation for predictive maintenance using kalman filter on a DC motor," Reliab. Eng. Syst. Saf., vol 75, pp. 103-111, 2002.

[17] R. David and H. Alla, "Petri nets for modeling of dynamic systems-A survey," Automatica, vol. 30, no. 2, pp. 175-202, 1994.

[18] G. S. Hura and J. W. Atwood, "The use of petri nets to analyze coherent fault trees," IEEE Trans. Rel., vol. 37, no. 5, pp. 469-474, 1988.
[19] W. G. Schneeweiss, Petri Net for Reliability Modeling: Lilole-Verlag, 1999.

[20] S. K. Yang and T. S. Liu, "Failure analysis for an airbag inflator by petri nets," Qual. Reliab. Eng. Int., vol. 13, pp. 139-151, 1997.

[21] T. Kumamaru, T. Utsunomiya, and Y. Yamada, "A fault diagnosis system for district heating and cooling facilities," in Proc. IECON'1991, Int Conf. on Industrial Electronics, Control, and Instrumentation, 1991, pp. 131-136.

[22] A. W. Shaw, Logic Circuit Design: Fort Worth Saunder College Publishing, 1993.

[23] Foundation Series Quick Start Guide, Ver. F1.4, The Programmable Logic Company, Xilinx, 1998.

[24] Hardware User Guide, The Programmable Logic Company, Xilinx, 1998.

S. K. Yang was born in Taiwan. He received the B.S. in 1982 and the M.S in 1985 in Automatic Control Engineering from Feng Chia University, Taiwan. From 1985 to 1991, he was an Assistant Researcher and Instrumentation System Engineer of the Flight Test Group, Aeronautic Research Laboratory, Chung Shan Institute of Science and Technology, Taiwan. Since 1991, he has been with the Department of Mechanical Engineering at National Chin Yi Institute of Technology, Taiwan, where he is an associate professor. He received the Ph.D. in 1999 in Mechanical Engineering from National Chiao Tung University, Taiwan. His research interests are in reliability, data acquisition, and automatic control. 